\title{
Optimum Relay Location in Cooperative Communication Networks with Single AF Relay
}

\author{
Lei Xu, Hong-Wei Zhang, Xiao-Hui Li*, Xian-Liang Wu \\ The Key Laboratory of Intelligent Computing and Signal Processing, Ministry of Education, \\ Anhui University, Hefei, China \\ E-mail:xhli@ahu.edu.cn \\ Received December 12, 2010; revised January 28, 2011; accepted February 3, 2011
}

\begin{abstract}
In recent years cooperative diversity has been widely used in wireless networks. In particular, cooperative communication with a single relay is a simple, practical technology for wireless sensor networks. In this paper, we analyze several simple network topologies. Under the condition of equal power allocating, the optimum relay location of each network topology are respectively made sure by using symbol error rate (SER) formula. And these types of topologies are compared, the analysis results show that, linear network topology has the best system performance, the system performance of isosceles triangle topology is better than that of equilateral triangle topology.
\end{abstract}

Keywords: Relay Location, Cooperative Communication, AF (Amplify and Forward), Topology

\section{Introduction}

Multiple antennas transmitting diversity technology has been investigated based on their ability to combat fading induced by multi-path of the channel, and has been widely used in wireless communication system. However, the deployment of antenna array on mobile terminal is difficult due to the size and power limitation. In order to solve this problem a new mode of gaining transmit diversity called cooperative diversity has been proposed and widely studied [1]. The terminals share their antennas and other resources to create a "virtual array" through distributed transmission and signal processing [2].

Compared with the multi-relay cooperative communication, single-relay cooperative communication has more practical value for wireless sensor networks [3]. The research for single-relay cooperative communication network topology has great significance for improving the performance of cooperative systems. Paper [4] has pointed out that, the location of relay has an impact on the performance of the system. However, the analytic network topology is single and has not given the theoretical basis of the optimal relay location. In this paper, what is mainly concerned is that, in the uncoded cooperative communication system, the position of relay is how to affect the SER performance of the system. According to the SER formula of single-relay cooperative communication system [5], in several equal-power allocated simple network topologies, the optimal relay location is trying to be gotten. The comparison system performance among these types of network topologies will be done.

\section{System Model}

The block diagram of single-relay cooperative communication system is shown as Figure 1.

The mechanism of single-relay cooperative communication is divided into two stages. Both stages use the orthogonal channel, such as TDMA, FDMA, or CDMA. In the first phase, source node sends symbols to the destination node; relay node receives the symbols sent from source node at the same time. The received symbols $y_{s, d}$ and $y_{s, r}$ at destination node and relay node, respectively, can be written as

$$
\begin{aligned}
& y_{s, d}=\sqrt{P_{1}} h_{s, d}[n] \cdot x_{s}[n]+z_{s, d}[n] \\
& y_{s, r}=\sqrt{P_{1}} h_{s, r}[n] \cdot x_{s}[n]+z_{s, r}[n]
\end{aligned}
$$

In which $x_{s}[n]$ is the transmitted information; $P_{1}$ is the transmitted power at the source node; $h_{s, d}$ is the channel coefficients from source node to destination node, which can be written as $h_{s, d}[n]=d_{s, d} \cdot a_{s, d}[n], a_{s, d}[n]$ can be modeled as a zero mean, complex Gaussian random 


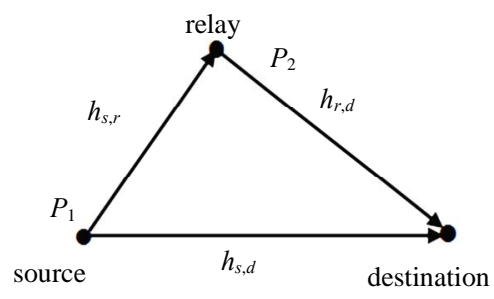

Figure 1. Block diagram of single-relay cooperative communication system.

variable with variances $\delta_{s, d}^{2}$, the path loss $d_{s, d}$ (assuming a plane-earth model) is proportional to $R_{s, d}^{-2}$, $R_{s, d}$ is the distance between source node and destination node. $h_{s, r}$ is the channel coefficients from source node to relay node, which can be written as $h_{s, r}[n]=d_{s, r} \cdot a_{s, r}[n]$, $a_{s, r}[n]$ can be modeled as a zero-mean, complex Gaussian random variables with variances $\delta_{s, r}^{2}$, the path loss $d_{s, r}$ (assuming a plane-earth model) $\mathrm{s}$ proportional to $R_{s, r}^{-2}, R_{s, r}$ is the distance between source node and relay node. $z_{s, d}[n]$ and $z_{s, r}[n]$ are additive white Gaussian noise.

In the second phase, relay node amplifies the received signal and forwards it to destination node with transmitted power $P_{2}$. The received symbols at the destination can be written as

$$
y_{r, d}=\frac{\sqrt{P_{2}}}{\sqrt{P_{1}\left|h_{s, r}\right|^{2}+N_{0}}} h_{r, d}[n] \cdot y_{s, r}+z_{r, d}[n]
$$

where $h_{r, d}$ is the channel coefficients from relay node to destination node, which can be written as $h_{r, d}[n]=$ $d_{r, d} \cdot a_{r, d}[n], a_{r, d}[n]$ can be modeled as a zero-mean, complex Gaussian random variables with variances $\delta_{r, d}^{2}$, the path loss $d_{r, d}$ (assuming a plane-earth model) is proportional to $R_{r, d}^{-2}, R_{r, d}$ is the distance between source node and relay node. $z_{r, d}[n]$ is additive white Gaussian noise.

Assuming the antenna modes used by source node, destination and relay node in the system are omni antennas. The power of transmitted symbols $x_{s}[n]$ is normalized. $\delta_{s, d}^{2}, \delta_{s, r}^{2}$ and $\delta_{r, d}^{2}$ are set to 1 .

\section{Theoretical Analysis}

THEOREM I: If all of the channel links $h_{s, d}, h_{s, r}$, $h_{r, d}$ are available, i.e., $\delta_{s, d}^{2} \neq 0, \delta_{s, r}^{2} \neq 0$ and $\delta_{r, d}^{2} \neq 0$, then when $P_{1} / N_{0}$ and $P_{2} / N_{0}$ go to infinity, the SER of the AF systems with M-PSK or M-QAM modulation can be tightly approximated as

$$
P_{s} \approx \frac{B N_{0}^{2}}{b^{2}} \cdot \frac{1}{P_{1} \delta_{s, d}^{2}}\left(\frac{1}{P_{1} \delta_{s, r}^{2}}+\frac{1}{P_{2} \delta_{r, d}^{2}}\right)
$$

where, for M-PSK signal, $b=b_{P S K}$ for M-PSK and [5]

$$
B=\frac{3(M-1)}{8 M}+\frac{\sin \frac{2 \pi}{M}}{4 \pi}-\frac{\sin \frac{4 \pi}{M}}{32 \pi}
$$

In which $M=4, b=1$, and for QPSK modulation adopted in this paper. So,

$$
B=\frac{9}{32}+\frac{1}{4 \pi}
$$

$N_{0}$ is the power value of additive white Gaussian noise for each channel.

When $P_{1}=P_{2}=P / 2, P$ is the fixed total transmission power $P=P_{1}+P_{2}$. Define

$$
\begin{array}{r}
\gamma_{1}=\frac{P_{1}}{N_{0}}, \gamma_{2}=\frac{P_{2}}{N_{0}}, \gamma=\frac{P}{N_{0}}, \text { so } \gamma_{1}=\gamma_{2}=\frac{1}{2} \gamma . \\
\overline{\gamma_{1}}=\frac{P_{1}\left|h_{s, d}\right|^{2}}{N_{0}}=\frac{P_{1} \delta_{s, d}^{2} R_{s, d}^{-4}}{N_{0}}=\gamma_{1} \delta_{s, d}^{2} R_{s, d}^{-4}=\frac{1}{2} \gamma \delta_{s, d}^{2} R_{s, d}^{-4} \\
\overline{\gamma_{2}}=\frac{P_{2}\left|h_{r, d}\right|^{2}}{N_{0}}=\frac{P_{2} \delta_{r, d}^{2} R_{r, d}^{-4}}{N_{0}}=\gamma_{2} \delta_{r, d}^{2} R_{r, d}^{-4}=\frac{1}{2} \gamma \delta_{r, d}^{2} R_{r, d}^{-4} \\
\overline{\gamma_{3}}=\frac{P_{1}\left|h_{s, r}\right|^{2}}{N_{0}}=\frac{P_{1} \delta_{s, r}^{2} R_{s, r}^{-4}}{N_{0}}=\gamma_{1} \delta_{s, r}^{2} R_{s, r}^{-4}=\frac{1}{2} \gamma \delta_{s, r}^{2} R_{s, r}^{-4}
\end{array}
$$

With channel model, (4) is transformed as follows through the use of (6), (7) and (8).

$$
P_{s} \approx \frac{4 B}{\gamma R_{s, d}^{-4}}\left(\frac{1}{\gamma R_{s, r}^{-4}}+\frac{1}{\gamma R_{r, d}^{-4}}\right)
$$

\section{Topology Models Analysis}

\subsection{Equilateral Triangle Topology}

Because $R_{s, d}=R_{s, r}=R_{r, d}=R$, (9) is transformed as follows.

$$
P_{s}=\frac{8 B}{\left(\gamma R^{-4}\right)^{2}}
$$

The transform relation between bit error rate (BER) $P_{b}$ and SER $P_{s}$ is as follows.

$$
P_{b}=1-\sqrt{1-P_{s}}
$$

So (10) can be transformed to (12)

$$
P_{b}=1-\sqrt{1-P_{s}}=1-\sqrt{1-\frac{8 B}{\left(\gamma R^{-4}\right)^{2}}}
$$

When $R=1$, we can get

$$
\begin{gathered}
P_{s} \approx \frac{8 B}{\gamma^{2}} \\
P_{b}=1-\sqrt{1-P_{s}}=1-\sqrt{1-\frac{8 B}{\gamma^{2}}}
\end{gathered}
$$


The comparison graphic of BER between simulation and the asymptotically tight approximation is shown as Figure 2.

The comparison graphic of BER among $R=2, R=1.5$, $R=1, R=0.7, R=0.5$ is shown as Figure 3 .

Some conclusions can be obtained from the analysis of Figure 3.

1) When $R>1$, the BER increases as $R$ increases.

2) When $R=1$, the BER only matters with $\gamma$. In this situation, the path attenuation is not considered.

3) When $R<1$, the performance of BER is better than that of the situation $R>1$, the BER increases as $R$ increases.

There is a constraint condition for $R, R \geq R_{\min } . R_{\min }$ is used to ensure the space nonrelated performance of antenna among source node, destination node and relay node.

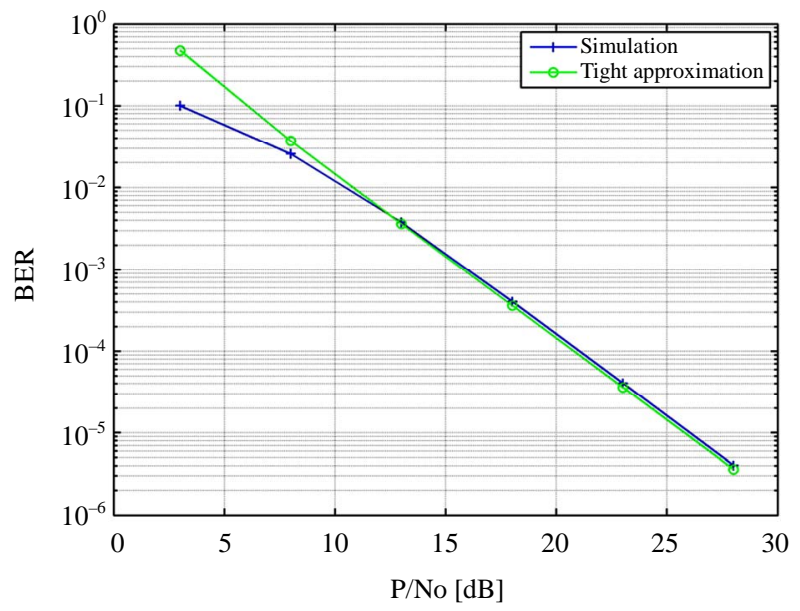

Figure 2. The comparison of BER between simulation and the asymptotically tight approximation.

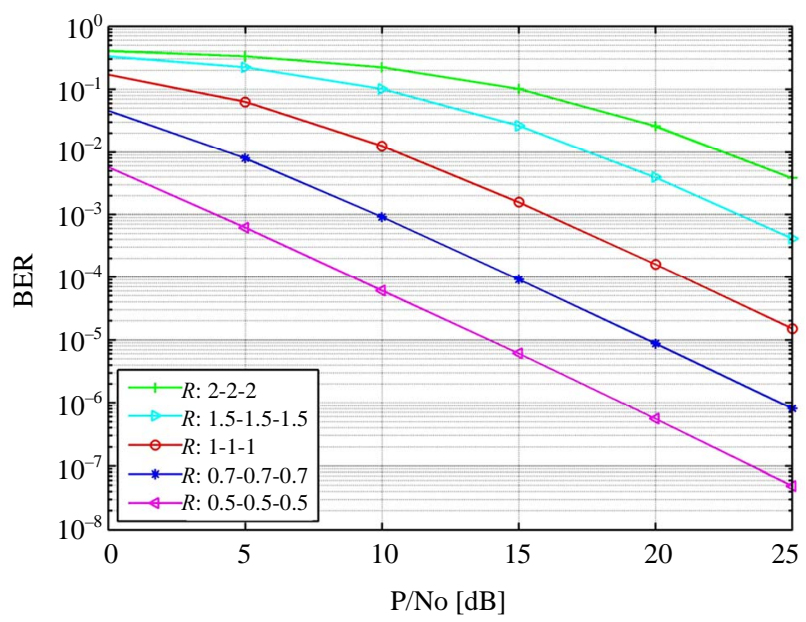

Figure 3. The comparison of BER among $R=2, R=1.5, R=$ $1, R=0.7, R=0.5$.

\subsection{Isosceles Triangle Topology}

Isosceles triangle topology is shown as Figure 4.

When $R_{s, d}=R_{s, r}=R$,

$$
R_{r, d}=R \sqrt{2(1-\cos \alpha)}, \alpha \in\left[-\frac{\pi}{3}, 0\right) \cup\left(0, \frac{\pi}{3}\right]
$$

Because of the symmetry of the topology, the situation of $\alpha \in(0, \pi / 3]$ is mainly researched.

When $R_{s, d}=R_{r, d}=R$,

$$
R_{s, r}=R \sqrt{2(1-\cos \beta)}, \alpha \in\left[-\frac{\pi}{3}, 0\right) \cup\left(0, \frac{\pi}{3}\right]
$$

Because of the symmetry of the topology, the situation of $\beta \in(0, \pi / 3]$ is mainly researched.

When $\alpha=\beta=\pi / 3$, the isosceles triangle topology becomes the equilateral triangle topology.

When $\alpha=\pi / 4$ and $R=1, R_{r, d}=0.765367$. When $\beta=\pi / 4$ and $R=1, R_{s, r}=0.765367$. The comparison graphic of BER between these two type topologies is shown as Figure 5.

Through the analysis of Figure 5, we can see that, topology a and topology b have the symmetry structure and their BER performance also have the symmetry. If $\alpha=\beta$, then

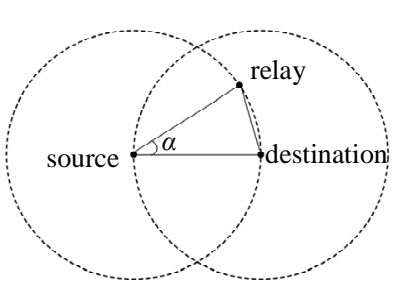

(a) $R_{s, d}=R_{s, r}=R$

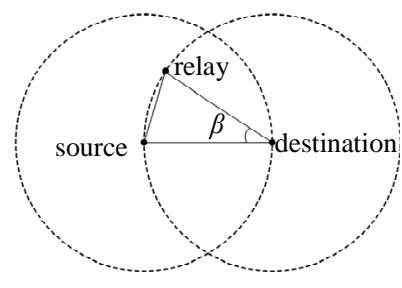

(b) $R_{s, d}=R_{r, d}=R$
Figure 4. Isosceles triangle topology.

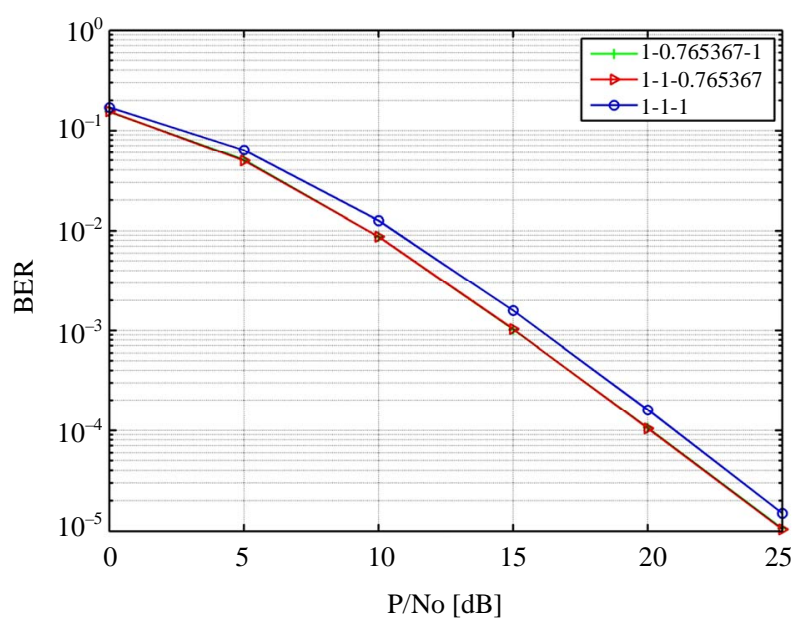

Figure 5. The comparison of BER with different $\boldsymbol{R}_{s, d}-\boldsymbol{R}_{s, r}-$ $\boldsymbol{R}_{r, d}$. 
$P_{s} \mid$ Isosceles Triangle a $=P_{s} \mid$ Isosceles Triangle $\mathrm{b}$

$P_{s} \mid$ Isosceles Triangle a $\approx \frac{4 B}{\gamma}\left(\frac{1}{\gamma}+\frac{1}{\gamma(\sqrt{2(1-\cos \alpha)})^{-4}}\right)$

From (16), the SER performance of the isosceles triangle a topology will be improved if $\alpha$ decreases. There is a constraint condition for $\alpha, \alpha \geq \alpha_{\min }, \alpha_{\text {min }}$ is used to ensure the space non-related performance of antenna between source node and relay node.

$$
P_{s} \mid \text { Isosceles Triangle } \mathrm{b} \approx \frac{4 B}{\gamma}\left(\frac{1}{\gamma(\sqrt{2(1-\cos \beta)})^{-4}}+\frac{1}{\gamma}\right)
$$

From (17), the SER performance of the isosceles triangle $\mathrm{b}$ topology will be improved if $\beta$ decreases. There is a constraint condition for $\beta, \beta \geq \beta_{\min }, \beta_{\text {min }}$ is used to ensure the space nonrelated performance of antenna between source node and relay node.

\subsection{Linear Topology}

Linear Topology is shown as Figure 6.

Assuming $R_{s, d}$ is a fixed value, $R_{s, r}=\rho R_{s, d}$. When $\rho \in(0,1), R_{r, d}=R_{s, d}(1-\rho)$. The process of determining the optimal location of relay is as follows.

$$
P_{s} \mid \text { Linear } \approx \frac{4 B}{\gamma R_{s, d}^{-4}}\left(\frac{1}{\gamma\left(\rho R_{s, d}\right)^{-4}}+\frac{1}{\gamma\left[(1-\rho) R_{s, d}\right]^{-4}}\right)
$$

The second derivative of (18) with respect to $\rho$ is

$$
\frac{4 B}{\gamma^{2} R_{s, d}^{-8}}\left[12 \rho^{2}+12(1-\rho)^{2}\right]
$$

(19) $\geq 0$, so the $\rho$ corresponding to the minimum value of (18) is existing.

Make the first derivative of (18) with respect to $\rho$ is equal to 0 .

$$
\frac{4 B}{\gamma^{2} R_{s, d}^{-8}}\left[4 \rho^{3}-4(1-\rho)^{3}\right]=0
$$

The root satisfying the constraint condition $\rho \in(0,1)$ is 0.5 .

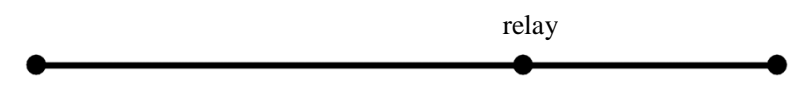

source

destination

Figure 6. Linear topology.
Make $R_{s, d}$ equal to 1 , the comparison graphic of BER with different $R_{s, d}-R_{s, r}-R_{r, d}$ is shown as Figure 7.

We can see that, if the linear topology structures are symmetric, then the BER performance also have the symmetry.

\section{The Comparison of Performance among Topologies}

Make $R_{s, d}=1$, the comparison graphic of BER among equilateral triangle topology, isosceles triangle topology b $\left(\beta=\pi / 6, R_{s, r}=0.517638\right)$, linear topology $(\rho=0.5)$ is shown as Figure 8.

We can see that, the BER performance of linear topology $(\rho=0.5)$ is the best, the BER performance of isosceles triangle topology $\mathrm{b}\left(\beta=\pi / 6, R_{s, r}=0.517638\right)$ is better than that of equilateral triangle topology. But there are also some questions existing.

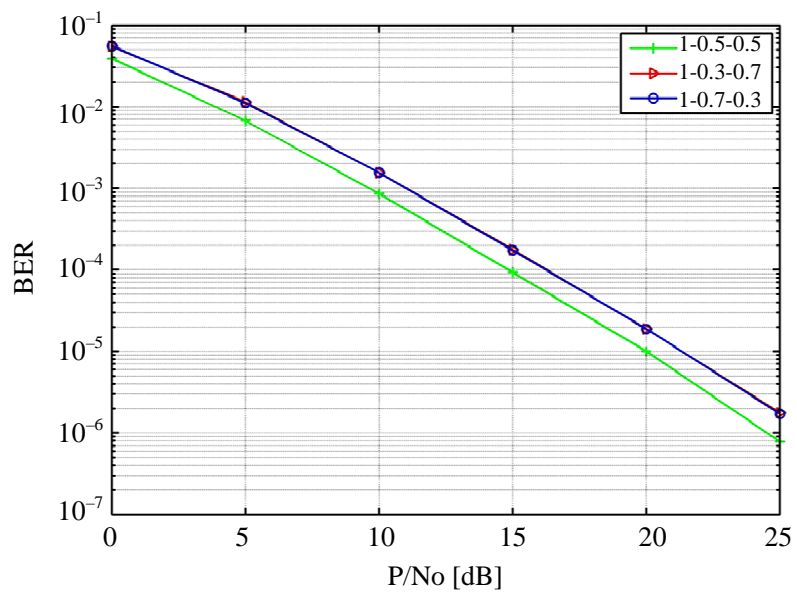

Figure 7. The comparison of BER with different $R_{s, d}-R_{s, r}$ $\boldsymbol{R}_{r, d}$

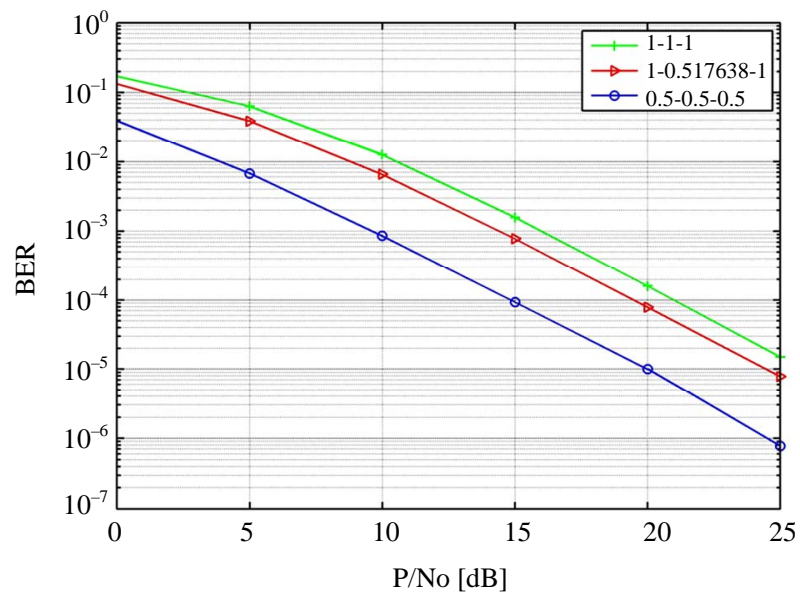

Figure 8. The comparison of performance among topologies. 
Question 1): Is the BER performance of isosceles triangle topology b with optimal relay location always better than that of equilateral triangle topology?

From (13), we can get

$$
P_{s} \mid \text { Equilateral Triangle }=\frac{4 B}{\gamma}\left(\frac{1}{\gamma}+\frac{1}{\gamma}\right)
$$

compare (21) to (17), because $\beta \in(0, \pi / 3]$,

$\cos \beta \in(1,1 / 2]$ and $\sqrt{2(1-\cos \beta)} \in(0,1]$,

$$
P_{s} \mid \text { Isosceles triangle } \mathrm{b} \leq P_{s} \mid \text { Equilateral triangle }
$$

when $\beta=\pi / 3$, the equal mark exists.

Question 2): Is the BER performance of linear topology with optimal relay location always better than that of isosceles triangle topology b with optimal relay location?

The SER formula of isosceles triangle topology b with optimal relay location is as follows.

$$
\begin{aligned}
\left.P_{s}\right|_{\text {min }} ^{\text {Isosceles Triangle b }} & =\lim _{\beta \rightarrow 0} \frac{4 B}{\gamma}\left(\frac{1}{\gamma(\sqrt{2(1-\cos \beta)})^{-4}}+\frac{1}{\gamma}\right)_{(22)} \\
& =\frac{4}{\gamma^{2}} \cdot B=\frac{4}{\gamma^{2}} \cdot 0.360827
\end{aligned}
$$

The SER formula of linear topology with optimal relay location is as follows.

$$
P_{s} \mid \begin{aligned}
& \text { Linear } \\
& \rho=0.5
\end{aligned}=\frac{4 B}{\gamma^{2}}\left[\rho^{4}+(1-\rho)^{4}\right]=\frac{4}{\gamma^{2}} \cdot 0.0451034
$$

Compare (22) to (23), we can get

$$
\left.P_{s}\right|_{\operatorname{lin}} ^{\text {Isosceles Trangle b }}>P_{s} \mid \begin{aligned}
& \text { Linear } \\
& \rho=0.5
\end{aligned}
$$

In summary, the BER performance of linear topology with optimal relay location is the best, the BER performance of isosceles triangle topology b with optimal relay location is better than that of equilateral triangle topology.

\section{Conclusions}

In this paper, under the condition of equal power allocating, the optimal relay location of several type topologies is determined. The research of the optimal relay location provides a good data reference for getting the best BER performance in the actual topology of powerlimited network. The cooperative communication networks have the features that, if the topology structures are symmetric, then the BER performance also has the symmetry. Under certain conditions, linear topology is the best by comparison. In order to get the better BER performance, the use of cooperative positioning relay is a good choice. Optimum power allocation techniques can also make the BER performance of the system with optimal relay location be well improved.

\section{Acknowledgements}

This work is supported by the National Nature Science Foundation of China (60972040), the Anhui provincial natural science foundation (11040606Q06), the key research project foundation of Anhui education department (KJ2009A57) and the Program of Superior Teacher Team in Anhui University of China (02203104).

\section{References}

[1] W. W. Liu, H. X. Sun, W. J. Shen and Y. H. Zhang, "Research of Cooperative Diversity Applied in OFDM System," Proceedings of International Conference on Intelligent Computation Technology and Automation, Changsha, 11-12 May 2010, pp. 1043-1046. doi:10.1109/ICICTA.2010.778

[2] J. N. Laneman, D. N. C. Tse and G. W. Wornell, “Cooperative Diversity in Wireless Network: Efficient Protocol and Outage Behavior," IEEE Transactions on Information Theory, Vol. 50, No. 12, December 2004, pp. 30623080. doi:10.1109/TIT.2004.838089

[3] C. Zhang, W. D. Wang, G. Wei and Y. Z. Li, "Power Efficient Cooperative Relaying with Node Location Information in Wireless Sensor Networks," Proceedings of International Conference on Intelligent Sensors, Sensor Networks and Information, Sydney, 15-18 December 2008, pp. 411-416.

[4] F. Lin, Q. H. Li, T. Luo and G. X. Yue, "Impact of Relay Location According to SER for Amplify-and-Forward Cooperative Communications,” 2007 IEEE International Workshop on Anti-counterfeiting, Security, Identification. Xiamen, 16-18 April 2007, pp. 324-327.

[5] K. J. R. Liu, A. K. Sadek, W. F. Su and A. Kwasinski, "Cooperative Communications and Networking," Cambridge University Press, New York, 2009, pp. 158-162. 